\title{
ON ALMOST-PRIMES IN ARITHMETIC PROGRESSIONS
}

\author{
By
}

\author{
Hiroshi Mikawa
}

\section{Introdnction.}

Let $P_{r}$ denote integers with at most $r$ prime factors counted according to multiplicity. In 1975, Y, Motohashi [9] showed that there exists a $P_{3}$ such that

$$
P_{3} \equiv a(\bmod q), \quad P_{3} \ll q(\log q)^{70}
$$

for any fixed non-zero integer $a$ and almost-all moduli $q$ with $(q, a)=1$. His argument based upon the weighted linear sieve and the Brun-Titchmarsh theorem on average, which are due to H.E. Richert [10] and C. Hooley [4], respectively. H. Iwaniec's fundamental works $[6,7]$, therefore, suggest possibilities of an improvement upon the above result. In this paper we present an estimation for $P_{2}$. We shall prove the following

THEOREM. Let $Q$ be a large parameter and $a$ be any fixed integer, $0<|a|$ $\leqq Q$. Then, except possibly for $O(Q / \log Q)$ moduli $q$ with $(q, a)=1$ and $Q<q \leqq 2 Q$, there exists a $P_{2}$ such that

$$
P_{2} \equiv a(\bmod q), \quad P_{2} \leqq \tau(a) q(\log q)^{7}
$$

where the implied O-constant is absolute and $\tau$ denotes the divisor function.

Our proof of Theorem is performed by a simple modification of the argument in our previous paper [8], in which the dual problem is considered. In fact, the numerical work in the main term from sieve estimate is identical. Succeeding to Hooley's investigation [4] we treat the remainder terms with the same manner as in [8]. Our main lemma (see Lemma 1 below) is weaker than E. Fouvry's works [1-3] in its scope; however, it will be found to be suitable for an application to the weighted sieve.

We use the standard notation in number theory. Especially, $\bar{r}$, used in either $r / s$ or congruence $(\bmod q)$, means that $\bar{r} r \equiv 1(\bmod s)$. $\sum_{x=1}^{y} *$ stands for the summation with restriction $(x, y)=1 . \quad n \sim N$ means $N \leqq N_{1}<n \leqq N_{2} \leqq 2 N$ for some $N_{1}$ and $N_{2}$. $\varepsilon$ denotes a small positive constant and the constants implied in the

Received June 19, 1989. 
symbols $\ll$ and $O$ may depend only on $\varepsilon$.

I would like to thank Professor S. Uchiyama for valuable comments and careful reading the original manuscript.

\section{Proof of Theorem.}

Firstly we state the inequality for $P_{2}$. This follows from Richert-ChenIwaniec's work, see [6]. For $q \leqq x$ and $(q, a)=1$, put

$$
\begin{aligned}
& \mathcal{A}=\{n: n \leqq x, n \equiv a(\bmod q),(n, a)=1\}, \\
& \mathcal{A}_{d}=\{n: n \in \mathcal{A}, n \equiv 0(\bmod d)\}, \\
& \mathcal{P}=\{p: p \nmid q\}, \quad \omega(d)= \begin{cases}1, & (d, a)=1 \\
0, & (d, a)>1,\end{cases}
\end{aligned}
$$

and

$$
r(\mathcal{A}, d)=\left|\mathcal{A}_{d}\right|-\frac{\phi(a)}{a} \frac{x}{q} \frac{\omega(d)}{d} .
$$

Let $\alpha, \alpha, v$ be the parameters such that

$$
\frac{1}{\alpha}<u<v, \quad \frac{2}{\alpha} \leqq v \leqq \frac{4}{u}, \quad u<3 .
$$

Write

$$
D=x^{\alpha}, \quad y=x^{1 / u}, \quad z=x^{1 / v} .
$$

Then the following inequality is valid.

$$
\begin{aligned}
& \Pi_{2}(x: q, a)=\left|\left\{P_{2}: P_{2} \leqq x, P_{2} \equiv a(\bmod q)\right\}\right| \\
& >\underset{\substack{p<2 \\
p \nmid q}}{\operatorname{II}}\left(1-\frac{\omega(p)}{p}\right) \frac{x}{q}\{C(\alpha, u, v)-E\}+\sum_{(d, q)=1} \lambda_{d} r(\mathcal{A}, d)-\sum_{z \leq p<y} \sum_{\substack{n \in \mathcal{A} \\
p^{2} \backslash n}} 1 \\
& =\Pi+E_{2}(q)+E_{1}(q), \quad \text { say, }
\end{aligned}
$$

where $C(\alpha, u, v)$ is some constant, $E$ is a very small quantity and the sequence $\left(\lambda_{d}\right)=\left(\lambda_{d}(D)\right)$ has the properties :

$$
\begin{aligned}
& \lambda_{d}=0 \quad \text { if } d \geqq D . \\
& \left|\lambda_{d}\right| \leqq \mu^{2}(d) .
\end{aligned}
$$

and for any $M>y, N>1, M N=D$,

$$
\lambda_{d}=\sum_{l \leqq(\log D)^{2}} \sum_{\substack{m \leq M \\ m n=d}} \sum_{\substack{n \leq N \\ m}} a_{m}(l, M, N) b_{n}(l, M, N)
$$

with $\left|a_{m}\right|,\left|b_{n}\right| \leqq 1$.

We next choose $\alpha=11 / 20-6 \varepsilon, 1 / u=1 / 2-8 \varepsilon, 1 / v=\alpha / 4+11 / 80-2 \varepsilon$, then the 
condition $(2.2)$ is satisfied and, for sufficiently large $x$ and small $\varepsilon$,

$$
v e^{-r}\{C(\alpha, u, v)-E\}>\frac{1}{200},
$$

which is caluculated in [8]. Hence

$$
\Pi>\frac{1}{200} \frac{x}{q \log x} .
$$

We turn to $E_{2}(q)$. We use the following lemma :

LEMMA 1. Let $2 Q<x, Q^{1 / 4} x^{-1 / 5}>x^{\varepsilon}$. If $M \leqq Q^{1 / 2} x^{-4 \varepsilon}$ and $N \leqq Q^{1 / 4} x^{-1 / 5}$, then

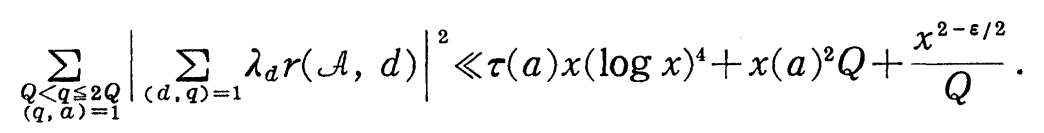

We postpone the proof of lemma 1 until the final sectien. Now we set $x=$ $\tau(a) Q(\log Q)^{7}, M=x^{1 / 2-5 \varepsilon}>y=x^{1 / u}=x^{1 / 2-8 \varepsilon}$ and $N=x^{11 / 20-\varepsilon}$, then $M \leqq Q^{1 / 2} x^{-4 \varepsilon}$ and $N \leqq Q^{1 / 4} x^{-1 / 5}$. Lemma 1 yields

$$
\begin{aligned}
\sum_{\substack{Q<q<\leq 2 Q \\
(q, a)=1}}\left|E_{2}(q)\right|^{2} & \ll \tau(a) x(\log x)^{4}+\tau(a)^{2} Q+\frac{x^{2-\varepsilon / 2}}{Q} \\
& \ll\left(\frac{x}{q}\right)^{2} Q \frac{\tau(a) Q(\log Q)^{4}}{x}+\left(\frac{x}{q}\right)^{2} Q^{1-\varepsilon / 2} \\
& \ll\left(\frac{x}{q}\right)^{2} Q(\log Q)^{-3}
\end{aligned}
$$

Moreover we have

$$
\begin{aligned}
\sum_{\substack{Q<q \leq 2 Q \\
(q, a)=1}}\left|E_{1}(q)\right| & \leqq \sum_{q} \sum_{x^{2 \varepsilon} \leq p \leq x^{1 / 2}} \sum_{\substack{a \leq n \leq x \\
n \equiv a(q) \\
p^{2} \mid n}} 1 \\
& \leqq \sum_{x^{2 \varepsilon} \leq p \leq x^{1 / 2}} \sum_{\substack{a<n \leq x \\
p^{2} \mid n}} \tau(n-a) \\
& \ll x^{\varepsilon} \sum_{p \geqq x^{2 \varepsilon}} \frac{x}{p^{2}} \\
& \ll x^{1-\varepsilon} \ll\left(\frac{x}{Q}\right) Q(\log Q)^{-2} .
\end{aligned}
$$

In conjunction with (2.3), (2.5), (2.6) and (2.7) we obtain

$$
\Pi_{2}\left(\tau(a) Q(\log Q)^{7}: q, a\right)>\frac{3 C x}{q \log x}+E_{1}(q)+E_{2}(q)
$$

where $C$ is a positive absolute constant and $E_{j}(q)(j=1,2)$ satisfy $(2.7)$ and $(2.6)$, respectively.

We proceed to the proof of Theorem. Put $x(t)=\tau(a) t(\log t)^{7}$ and $\mathcal{E}=$ $\left\{q: Q<q \leqq 2 Q,(q, a)=1, \Pi_{2}(x(q): q, a)=0\right\}$. We shall deduce $|\mathcal{E}| \ll Q(\log Q)^{-1}$, 
from which our Theorem will follow. Now, by (2.8), we have

$$
\Pi_{2}(x(q): q, a) \geqq \Pi_{2}(x(Q): q, a)>\frac{3 C x}{q \log x}+E_{1}(q)+E_{2}(q) .
$$

For all $q \in \mathcal{E}$ we then see that

$$
\left|E_{1}(q)\right|>\frac{C x}{q \log x} \text { or }\left|E_{2}(q)\right|>\frac{C x}{q \log x} .
$$

Hence

$$
\mathcal{E} \subset \mathcal{E}_{1} \cup \mathcal{E}_{2}
$$

where $\mathcal{E}_{j}=\left\{q: Q<q \leqq 2 Q,(q, a)=1,\left|E_{j}(q)\right|>\frac{C x}{q \log x}\right\}$. Furthermore,

$$
\left|\mathcal{E}_{j}\right|\left(\frac{C x}{2 Q \log x}\right)^{j}<\sum_{q \in \mathcal{E}_{j}}\left|E_{j}(q)\right|^{j} \leqq \sum_{\substack{Q<q \leq 2 Q \\(q, a)=1}}\left|E_{j}(q)\right|^{j}
$$

Combining this with (2.6), (2.7) and (2.9) we get

$$
\begin{aligned}
|\mathcal{E}| & \leqq \sum_{j=1,2}\left(\frac{Q \log Q}{x}\right)^{j} \cdot\left(\frac{x}{Q}\right)^{j} Q(\log Q)^{-j-1} \\
& \ll Q(\log Q)^{-1}
\end{aligned}
$$

as required.

\section{Proof of Lemma 1.}

In this section we follow the argument of [4] with a minor modification. We use the following elementary lemma: If $(c, d)=1$, then

$$
\sum_{\substack{A<m \leq B \\ m=e(d) \\(m, c)=1}}^{\sum} 1=\frac{\phi(c)}{c} \frac{B-A}{d}+O(\tau(c))
$$

By the definition (2.1) of $r(\mathcal{A}, d)$ we have

$$
\sum_{(d, q)=1} \lambda_{d} r(\mathcal{A}, d)=\sum_{\substack{a \leq n \leq x \\ n \equiv a(q) \\(n, a)=1}}\left(\sum_{\substack{d \backslash n \\(d, q)=1}} \lambda_{d}\right)-\frac{\phi(a)}{a} \frac{x-a}{q}\left(\sum_{(d, a q)=1} \frac{\lambda_{d}}{d}\right) .
$$

By (3.1),

$$
\begin{aligned}
& \sum_{\substack{(q, a)=1 \\
Q<q \leq Q}}\left|\sum_{(d, q)=1} \lambda_{d} r(\mathcal{A}, d)\right|^{2} \\
& \ll \sum_{\substack{(q, a)=1 \\
Q<q \leq Q}}\left|\sum_{\substack{a \leq n \leq x \\
n=a(q) \\
(n, a)=1}}\left(\sum_{\substack{d, n \\
(d, q)=1}} \lambda_{d}\right)-\left(\sum_{(d, a q)=1} \frac{\lambda_{d}}{d}\right) \sum_{\substack{a \leq n \leq x \\
n \leq n \\
(n, a)=1}} 1\right|^{2}+\tau(a)^{2} Q \\
& =W-2 V+U+\tau(a)^{2} Q, \quad \text { say. }
\end{aligned}
$$

Firstly we consider $W$. 


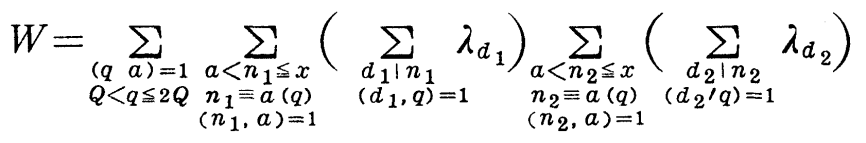

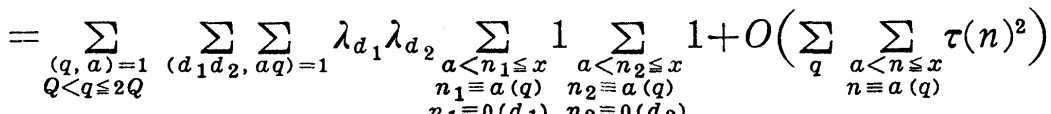

$$
\begin{aligned}
& \begin{array}{ll}
n_{1} \equiv 0\left(d_{1}\right) & n_{2} \equiv 0\left(d_{2}\right) \\
\left(n_{1}, a\right)=1 & \left(n_{2}, a\right)=1
\end{array}
\end{aligned}
$$

We express the congruent condition $n_{1} \equiv a(q), n_{2} \equiv a(q)$ as $n_{1}=a+q l_{1}, n_{2}=a+q l_{2}$. Then, the condition for $l_{1}$ and $l_{2}$ is

$$
l_{1} \neq l_{2} \leqq \frac{x-a}{q}, \quad\left\{\begin{array}{l}
a+q l_{1} \equiv 0\left(d_{1}\right) \\
a+q l_{2} \equiv 0\left(d_{2}\right)
\end{array}, \quad\left(l_{1} l_{2}, a\right)=1 .\right.
$$

Since $\left(l_{1} l_{2}, a\right)=1,\left(l_{1}, d_{1}\right)=\left(l_{2}, d_{2}\right)=1$. Changing the order of summation, we have

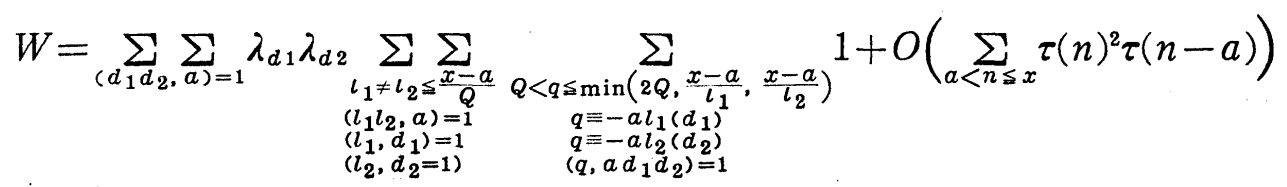

We write $L=(x-a) / Q$ and $Q^{\prime}=Q^{\prime}\left(l_{1}, l_{2}\right)=\min \left(2 Q,(x-a) / l_{1},(x-a) / l_{2}\right)$. The congruences are soluble iff $l_{1} \equiv l_{2}\left(\left(d_{1}, d_{2}\right)\right)$ and expressed as one congruence

$$
q \equiv b\left(\left[d_{1}, d_{2}\right]\right),\left\{\begin{array}{l}
b \equiv-a \bar{l}_{1}\left(d_{1}\right) \\
b \equiv-a \bar{l}_{2}\left(d_{2} *\right), \quad d_{2}^{*}=d_{2} /\left(d_{1}, d_{2}\right) .
\end{array}\right.
$$

This congruence then absorbs the condition $\left(q, d_{1} d_{2}\right)=1$. Hence

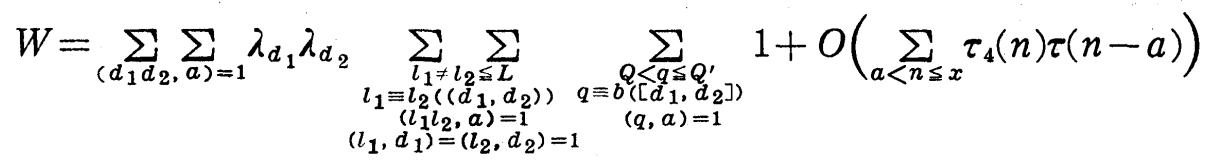

It is expected, by (3.1), that the innermost sum is approximately equal to

Thus,

$$
\frac{\phi(a)}{a} \frac{Q^{\prime}-Q}{\left[d_{1}, d_{2}\right]}
$$

where

$$
W=W_{0}+R+O\left(x(\log x)^{4}\right)
$$

$$
W_{0}=\sum_{\left(d_{1} d_{2}, a\right)=1} \frac{\lambda_{d_{1}} \lambda_{d_{2}}}{\left[d_{1}, d_{2}\right]} \frac{\phi(a)}{a} \cdot \sum_{\substack{l_{1} \neq l_{2} \leq L \\ l_{1} \equiv l_{2}\left(d, d_{1}\right) \\\left(l_{1}, a d_{1}\right)=\left(l_{2}, a d_{2}\right)=1}}\left(\min \left(2 Q, \frac{x-a}{l_{1}}, \frac{x-a}{l_{2}}\right)-Q\right)
$$

and

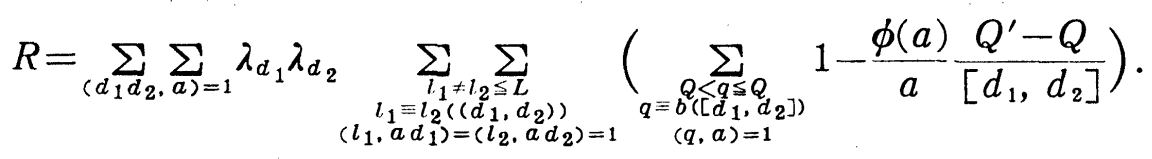


In section 5 we shall estimate $R$. We proceed to consider $W_{\mathbf{0}}$. We perform the summation over $l_{1}$ and $l_{2}$. Write

$$
\sum_{l_{1} \neq l_{2}}=\sum_{l_{1}<l_{2}}+\sum_{l_{1}>l_{2}}
$$

Then,

$$
\sum_{l_{1}<l_{2}}=\sum_{\substack{l_{2} \leq L \\\left(l_{2}, a d_{2}\right)=1}}\left(\min \left(2 Q, \frac{x-a}{l_{2}}\right)-Q\right) \sum_{\substack{\left.l_{1} \sum l_{2} \\ l_{1} \equiv l_{1}\left(l_{1}, d_{2}\right)\right) \\\left(l_{1}, a d_{1} l=1\right.}} 1
$$

By (3.1), the inner sum is equal to

$$
\begin{aligned}
\sum_{\substack{\left.l_{1}<l_{2}, d_{2}\right) \\
l_{1} \equiv l_{2}\left(\left(d_{1}, d_{2}\right)\right) \\
\left(l_{1}, a d_{1} *\right)=1}} 1=\frac{\phi\left(a d_{1} *\right)}{a d_{1}^{*}} \frac{l_{2}}{\left(d_{1}, d_{2}\right)}+O\left(\tau\left(a d_{1} *\right)\right) \\
=\frac{1}{\phi\left(\left(d_{1}, d_{2}\right)\right)} \sum_{\substack{\left.l_{1}<l_{2} \\
l_{1}, a d_{1}\right)=1}} 1+O\left(\tau\left(a d_{1}\right)\right),
\end{aligned}
$$

whence

Similarly,

$$
\sum_{l_{1}<l_{2}}=\frac{1}{\phi\left(\left(d_{1}, d_{2}\right)\right)} \sum_{\substack{l_{2} \leq L \\\left(l_{2}, a d_{2}\right)=1}}\left(\min \left(2 Q, \frac{x-a}{l_{1}}\right)-Q\right) \sum_{\substack{l_{1}<l_{2} \\\left(l_{1}, a d_{1}\right)=1}} 1+O\left(x\left(a d_{1}\right) x\right)
$$

$$
\sum_{l_{1}>l_{2}}=\frac{1}{\phi\left(\left(d_{1}, d_{2}\right)\right)} \sum_{\substack{l_{1} \leq L \\\left(l_{1}, a d_{1}\right)=1}}\left(\min \left(2 Q, \frac{x-a}{l_{1}}\right)-Q\right) \sum_{\substack{l_{2}<l_{1} \\\left(l_{2}, a d_{2}\right)=1}} 1+O\left(\tau\left(a d_{2}\right) x\right) .
$$

In conjunction with (3.5), (3.7) and (3.8) we have

$$
\begin{aligned}
& W_{0}=\sum_{\left(d_{1} d_{2}, a\right)=1} \frac{\lambda_{d_{1}} \lambda_{d_{2}}}{d_{1} d_{2}} \frac{\phi(a)}{a} \frac{\left(d_{1}, d_{2}\right)}{\phi\left(\left(d_{1}, d_{2}\right)\right)} \sum_{\substack{l_{1} \neq l_{2} \leq L \\
\left(l_{1}, a d_{1}\right)=\left(l_{2}, a d_{2}\right)=1}}\left(\min \left(2 Q, \frac{x-a}{l_{1}}, \frac{x-a}{l_{2}}\right)-Q\right) \\
& +O\left(\tau(a) x(\log x)^{3}\right) \\
& =W_{1}+O\left(\tau(a) x(\log x)^{3}\right), \quad \text { say. }
\end{aligned}
$$

Combining this with (3.4) we get

We turn to $V$.

$$
W=W_{1}+R+O\left(\tau(a) x(\log x)^{4}\right) .
$$

$$
\begin{aligned}
& V=\sum_{\substack{(q, a)=1 \\
Q<q \leq 2 Q}} \sum_{\substack{a<n_{1} \leq x \\
n_{1}=a(q) \\
\left(n_{1}, a\right)=1}}\left(\sum_{\substack{d, a n_{1} \\
\left(d_{1}, q\right)=1}} \lambda_{d_{1}}\right)\left(\sum_{\left(d_{2}, a q\right)=1} \frac{\lambda_{d_{2}}}{d_{2}}\right)_{\substack{a<n_{2} \leq x \\
n_{2}=a(q) \\
\left(n_{2}, a\right)=1}} 1 \\
& =\sum_{\substack{\left(q_{a}, a\right)=1 \\
Q<q \leq 2 Q}} \sum_{\substack{\left.1 \\
d_{2}, a q\right)=1}} \lambda_{d_{1}} \frac{\lambda_{d_{2}}}{d_{2}} \sum_{\substack{a<n_{1} \leq x \\
n_{1}=a(q) \\
n_{1}=0\left(d_{1}\right) \\
\left(n_{1}, a\right)=1}} 1 \sum_{\substack{a<n_{2} \leq x \\
n_{2}=a(q) \\
\left(n_{2}, a\right)=1 \\
n_{1} \neq n_{2}}} 1+O\left((\log x) \sum_{a<n \leq x} \tau(n) \tau(n-a)\right) .
\end{aligned}
$$


As before we write $n_{1}=a+q l_{1}$ and $n_{2}=a+q l_{2}$. Then the condition for $l_{1}$ and $l_{2}$ is

$$
l_{1} \neq l_{2} \leqq \frac{x-a}{q} . \quad a+q l_{1} \equiv 0\left(d_{1}\right), \quad\left\{\begin{array}{l}
\left(l_{1} l_{2}, a\right)=1 \\
\left(l_{1}, d_{1}\right)=1
\end{array} .\right.
$$

Changing the order of summation we have

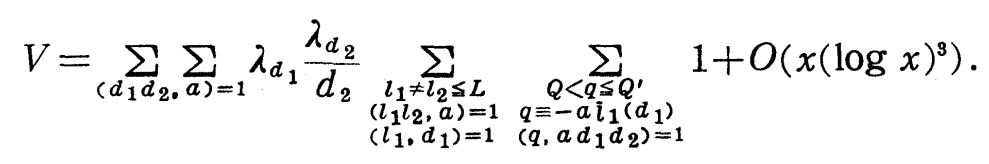

By (3.1) the innermost sum is equal to

$$
\sum_{\substack{Q<q \leq Q^{\prime} \\ q=a l_{1}\left(d_{1}\right) \\\left(q, a d_{2}^{*}\right)=1}} 1=\frac{\phi\left(a d_{2}^{*}\right)}{a d_{2}^{*}} \frac{\left(Q^{\prime}-Q\right)}{d_{1}}+O\left(\tau\left(a d_{2}^{*}\right)\right),
$$

whence

$$
\begin{aligned}
& V=\sum_{\left(d_{1} d_{2}, a\right)=1} \frac{\lambda_{d_{1}} \lambda_{d_{2}}}{d_{1} d_{2}} \frac{\phi\left(a d_{2}^{*}\right)}{a d_{2}^{*}} \sum_{\begin{array}{c}
\sum_{1 \neq l} \neq l_{2} \leq L \\
\left(1_{1} l_{2}(a)=1\right. \\
\left(l_{1}, d_{1}\right)=1
\end{array}}\left(Q^{\prime}-Q\right) \\
& +O\left(\tau(a)\left(\frac{x}{Q}\right)^{2} D(\log x)^{2}+x(\log x)^{3}\right)
\end{aligned}
$$

Next we carry out the summation over $l_{1}$ and $l_{2}$.

$$
\begin{aligned}
& \frac{\phi\left(d_{2}^{*}\right)}{d_{2}^{*}} \sum_{\substack{l_{1} \neq l_{2} \leq L \\
\left(l_{1} l_{2}, a\right)=1 \\
\left(l_{1}, a_{1}\right)=1}}\left(\min \left(2 Q, \frac{x-a}{l_{1}}, \frac{x-a}{l_{2}}\right)-Q\right) \\
& =\sum_{\substack{l_{2} \leq L \\
\left(l_{2}, a\right)=1}}\left(\min \left(2 Q, \frac{x-a}{l_{2}}\right)-Q\right) \frac{\phi\left(d_{2}^{*}\right)}{d_{2}^{*}} \sum_{\substack{l_{1}<l_{2} \\
\left(l_{1}, a d_{1}\right)=1}} 1 \\
& +\sum_{\substack{l_{1} \leq L \\
\left(l_{1}, a d_{1}\right)=1}}\left(\min \left(2 Q, \frac{x-a}{l_{1}}\right)-Q\right) \frac{\phi\left(d_{2}^{*}\right)}{d_{2}^{*}} \sum_{\substack{l_{2}<l_{1} \\
\left(l_{1}, a\right)=1}} 1 .
\end{aligned}
$$

(3.1) yields

and

$$
\frac{\phi\left(d_{2}^{*}\right)}{d_{2}^{*}} \sum_{\substack{l_{1}<l_{2} \\\left(l_{1}, a d_{1}\right)=1}} 1=\frac{\phi\left(\left[d_{1}, d_{2}\right]\right)}{\left[d_{1}, d_{2}\right]} \sum_{\substack{l_{1}<l_{2} \\\left(l_{1}, a\right)=1}} 1+O\left(\tau\left(a d_{1}\right)\right)
$$

Thus (3.11) is equal to

$$
\frac{\phi\left(d_{2}^{*}\right)}{d_{2}^{*}} \sum_{\substack{l_{2}<l_{1} \\\left(l_{2}, a\right)=1}} 1=\frac{\left(d_{1}, d_{2}\right)}{\phi\left(\left(d_{1}, d_{2}\right)\right)} \sum_{\substack{l_{2}<l_{1} \\\left(l_{2}, a d_{1}\right)=1}} 1+O\left(\tau\left(a d_{1}\right) \log x\right) .
$$

$$
\frac{\phi\left(\left[d_{1}, d_{2}\right]\right)}{\left[d_{1}, d_{2}\right]} \sum_{\substack{l_{1}<\sum_{2} \leq L \\\left(l_{1} l_{2}, a\right)=1}}\left(Q^{\prime}-Q\right)+\frac{\left(d_{1}, d_{2}\right)}{\phi\left(\left(d_{1}, d_{2}\right)\right)} \sum_{\substack{l_{2}<l_{1} \leq L \\\left(l_{1}, a d_{1}\right)=1 \\\left(l_{2}, a d_{2}\right)=1}}\left(Q^{\prime}-Q\right)+O\left(\tau\left(a d_{1}\right) \log x\right)
$$

Combining this with (3.10) we have 


$$
\begin{aligned}
& V=\sum_{\left(d_{1} d_{2}, a\right)=1} \frac{\lambda_{d_{1}} \lambda_{d_{2}}}{d_{1} d_{2}} \frac{\phi(a)}{a} \cdot\left(\frac{\phi\left(\left[d_{1}, d_{2}\right]\right)}{\left[d_{1}, d_{2}\right]} \sum_{\substack{l_{1}<l_{2} \leq L \\
\left(l_{1} l_{2}, a\right)=1}}\left(Q^{\prime}-Q\right)\right. \\
& \left.+\frac{\left(d_{1}, d_{2}\right)}{\phi\left(\left(d_{1}, d_{2}\right)\right)} \sum_{\substack{l_{2}<l_{1} \leq L \\
\left(l_{1}, a d 1\right)=1 \\
\left(l_{2}, a d_{2}\right)=1}}\left(Q^{\prime}-Q\right)\right) \\
& +O\left(\tau(a) x(\log x)^{4}+\tau(a)\left(\frac{x}{Q}\right)^{2} D(\log x)^{2}\right)
\end{aligned}
$$

Interchanging the role of $\left(d_{1}, l_{1}\right)$ with that of $\left(d_{2}, l_{2}\right)$, we may obtain the corresponding expression to (3.12). Hence

$$
2 V=U_{1}+W_{1}+O\left(\tau(a) x(\log x)^{4}+\tau(a)\left(\frac{x}{Q}\right)^{2} D(\log x)^{2}\right)
$$

where

$$
U_{1}=\sum_{\left(d_{1} d_{2}, a\right)=1} \frac{\lambda_{d_{1}} \lambda_{d_{2}}}{d_{1} d_{2}} \frac{\phi\left(a\left[d_{1}, d_{2}\right]\right)}{a\left[d_{1}, d_{2}\right]} \sum_{\substack{l_{1} \neq l_{2} \leq L \\\left(l_{1} l_{2}, a\right)=1}}\left(Q^{\prime}-Q\right) .
$$

Finaly we consider $U$. By the same argument as above, we have

$$
\begin{aligned}
& U=\sum_{\substack{(q, a)=1 \\
Q<q \leq Q Q}}\left(\sum_{\left(d_{1}, a q\right)=1} \frac{\lambda_{d_{1}}}{d_{1}}\right)\left(\sum_{\substack{\left(d_{2}, a q\right)=1 \\
\lambda_{2}}} \frac{\lambda_{d_{2}}}{d_{2}}\right)_{\substack{a<n_{1} \leq x \\
n_{1}=a(q) \\
\left(n_{1}, a\right)=1}} 1 \sum_{\substack{a<n_{2} \leq x \\
n_{2} \equiv a(q) \\
\left(n_{2}, a\right)=1}} 1
\end{aligned}
$$

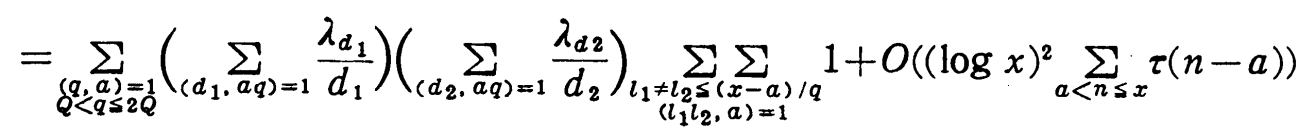

$$
\begin{aligned}
& =\sum_{\left(d_{1} d_{2}, a\right)=1} \sum_{\frac{\lambda_{d_{1}} \lambda_{d_{2}}}{d_{1} d_{2}}} \sum_{\substack{l_{1} \neq l_{2} \leq L \\
\left(l_{1} l_{2}, a\right)=1}} \sum_{\substack{Q<q \leq Q^{\prime} \\
\left(q, a\left[d_{1}, d_{2}\right]\right)=1}} 1+O\left(x(\log x)^{3}\right) \\
& =\sum_{\left(d_{1} d_{2}, a\right)=1} \sum_{a} \frac{\lambda_{d_{1}} \lambda_{d_{2}}}{d_{1} d_{2}} \sum_{\substack{l_{1} \neq l_{2} \leq L \\
\left(l_{1} l_{2}, a\right)=1}}\left(\frac{\phi\left(a\left[d_{1}, d_{2}\right]\right)}{a\left[d_{1}, d_{2}\right]}\left(Q^{\prime}-Q\right)+O\left(\tau\left(a\left[d_{1}, d_{2}\right]\right)\right)\right. \\
& +O\left(x(\log x)^{3}\right) \\
& =U_{1}+O\left(\tau(a)\left(\frac{x}{Q}\right)^{2}(\log x)^{4}+x(\log x)^{3}\right)
\end{aligned}
$$

In conjunction with (3.2), (3.9), (3.13) and (3.14) we get

$$
\begin{aligned}
& \sum_{\substack{Q<q \leq 2 Q \\
(q, a)=1}}\left|\sum_{(d, q)=1} \lambda_{d} r(\mathcal{A}, d)\right|^{2} \ll W_{1}+R-\left(U_{1}+W_{1}\right)+U_{1}+\tau(a) x(\log x)^{4} \\
&+\tau(a)\left(\frac{x}{Q}\right)^{2} D(\log x)^{2}+\tau(a)^{2} Q \\
& \leqq R+\tau(a) x(\log x)^{4}+\tau(a)^{2} Q+\left(\frac{x}{Q}\right)^{2} D x^{\varepsilon}
\end{aligned}
$$




\section{Auxiliary results.}

In the next section we use the following lemmas, see [5].

LEMMA 2. Let $\phi(t)=[t]-t+1 / 2$. For $H>2$, we have

$$
\psi(t)=\sum_{0<|h| \leqslant H} \frac{e(h t)}{2 \pi i h}+O\left(\min \left(1, \frac{1}{H\|t\|}\right)\right)
$$

where $e(x)=e^{2 \pi i x}$ and $\|x\|=\min _{n \in Z}|x-n|$. Moreover,

$$
\min \left(1, \frac{1}{H\|t\|}\right)=\sum_{h \in Z} C_{h} e(h t)
$$

with

$$
\left|C_{h}\right| \ll \min \left(\frac{\log H}{H}, \frac{H}{h^{2}}\right)
$$

LEMMA 3. We have, for any $\varepsilon>0$,

$$
\sum_{\substack { m \sim M \\
\begin{subarray}{c}{x \rightarrow \text { mod } \\
(m, . d)=1{ m \sim M \\
\begin{subarray} { c } { x \rightarrow \text { mod } \\
( m , . d ) = 1 } }\end{subarray}} e\left(l \frac{\bar{m}}{d}\right) \ll \tau(c)(l, d)^{1 / 2} d^{1 / 2+\varepsilon}\left(1+\frac{M}{d}\right) .
$$

\section{Proof of Lemma 1, continued.}

In this section we shall estimate $R$ by appealing to lemmas 3 and 4 . We begin with treating the condition $(q, a)=1$ by Moebius function.

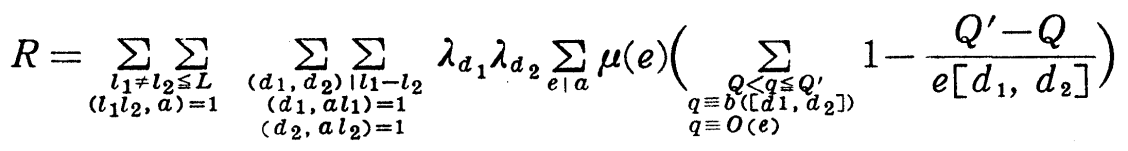

$$
\begin{aligned}
& =\sum_{e \mid a} \mu(a) \sum_{\substack{1 \\
l_{1} \neq l_{2} \leq L \\
\left(l_{1} l_{2}, a\right)=1}} \sum_{\substack{\left(d_{1}, d_{2}\right) l_{1}+l_{1}-l_{2} \\
\left(d_{1}, a l_{1}\right)=1 \\
\left(d_{2}, a l_{2}\right)=1}} \lambda_{d_{1}} \lambda_{d_{2}} \\
& \times\left\{\psi\left(\frac{Q^{\prime}}{e\left[d_{1}, d_{2}\right]}-\frac{b \bar{e}}{\left[d_{1}, d_{2}\right]}\right)-\psi\left(\frac{Q}{e\left[d_{1}, d_{2}\right]}-\frac{b \bar{e}}{\left[d_{1}, d_{2}\right]}\right)\right\} .
\end{aligned}
$$

We consider the argument in $\phi$-function. By the definition $(3.3)$ of $b\left(\bmod \left[d_{1}, d_{2}\right]\right)$ and the relalation, for $(m, n)=1$,

$$
\frac{\bar{m}}{n}+\frac{\bar{n}}{m} \equiv \frac{1}{m n} \quad(\bmod 1),
$$

we have 


$$
\begin{aligned}
-\frac{b \bar{e}}{\left[d_{1}, d_{2}\right]} & \equiv-\frac{b \overline{d_{2}^{*} e}}{d_{1}}-\frac{\overline{b \overline{d_{1} e}}}{\bar{d}_{2}^{*}} \\
& \equiv \frac{a l_{1} \bar{d}_{2}^{*} e}{d_{1}}+\frac{a \overline{l_{2} d_{1} e}}{\overline{d_{2}{ }^{*}}} \\
& \equiv \frac{a}{e}\left(\frac{\overline{d_{2}^{*} l_{1}}}{d_{1}}+\frac{\overline{d_{1} l_{2}}}{d_{2}{ }^{*}}\right) \\
& \equiv \frac{a}{e}\left(\frac{1}{d_{1} d_{2}{ }^{*} l_{1}}-\frac{\overline{d_{1}}}{d_{2}{ }^{*} l_{1}}+\frac{d_{1} l_{2}}{d_{2}{ }^{*}}\right) \quad(\bmod 1) .
\end{aligned}
$$

We write $l_{1}^{*}=l_{1} /\left(l_{1}, l_{2}\right), l_{2}^{*}=l_{2} /\left(l_{1}, l_{2}\right)$. When

$$
\begin{aligned}
& \left(l_{1}, l_{2}\right)={p_{1}}^{a_{1}} \cdots p_{r}{ }^{a_{r}} \\
& l_{1}{ }^{*}=p_{1}{ }^{b_{1}} \cdots p_{r}{ }^{b_{r}} p_{r+1}{ }^{a_{r+1}} \cdots{p_{s}}^{a_{s}} \\
& p_{i} \neq p_{j}(i \neq j), \quad a_{i} \geqq 1, \quad b_{i} \geqq 0,
\end{aligned}
$$

we define

$$
\begin{aligned}
& l_{1}^{* *}=p_{r+1}{ }^{a_{r+1}} \cdots p_{s}{ }^{a_{s}} \\
& l_{1}^{\#}={p_{1}}^{a_{1}+b_{1}} \cdots p_{r}{ }^{a_{r}+b_{r}} .
\end{aligned}
$$

Then it follows $\left(l_{1}^{* *}, l_{1}^{\#}\right)=1$ and $\left(d_{1} d_{2} *, l_{1}^{\#}\right)=1$. By (5.2),

$$
\frac{\bar{d}_{1}}{d_{2} l_{1}} \equiv \frac{\bar{d}_{1}}{d_{2} * l_{1}^{* *} \cdot l_{1}^{\#}} \equiv \frac{\overline{d_{1} l_{1}}}{d_{2} l_{1} l_{1}}+\frac{\overline{d_{1} d_{2}^{*} l_{1} * *}}{l_{1}^{\#}} \quad(\bmod 1) \text {. }
$$

Moreover,

$$
-\frac{\overline{d_{1} l_{1}}}{d_{2}{ }^{*} l_{1} * *}+\frac{\overline{d_{1} l_{2}}}{d_{2}{ }^{*}} \equiv \frac{k}{d_{2}{ }^{*} l_{1} * *}(\bmod 1)
$$

where $k=-{\overline{d_{1} l_{1}}}^{*}+\widehat{d_{1} l_{2} l_{1}} * *$ with $\widehat{d_{1} l_{2}} d_{1} l_{2} \equiv 1\left(\bmod d_{2} *\right)$. We then have, with a certain integer $n$,

$$
\begin{aligned}
d_{1} l_{1}{ }^{*} l_{2} * k & =-{\overline{d_{1} l_{1}}}^{\#} d_{1} l_{1}^{\#} l_{2} *+\widehat{d_{1} l_{2}} d_{1} l_{2} * l_{1}{ }^{*} l_{1} * * \\
& \equiv-l_{2} *+\widehat{d_{1} l_{2}} d_{1} l_{2} l_{1} * \\
& \equiv-l_{2} *+\left(1+n d_{2} *\right) l_{1} * \\
& \equiv l_{1} *-l_{2} * \quad\left(\bmod d_{2} * l_{1} * *\right) .
\end{aligned}
$$

Since $\left(d_{1} l_{1} l_{2}{ }^{*}, d_{2} * l_{1} * *\right)=1$,

$$
k \equiv\left(l_{1} *-l_{2} *\right) \overline{d_{1} l_{1}{ }^{*} l_{2}{ }^{*}} \quad\left(\bmod d_{2} * l_{1} * *\right) .
$$

The condition $\left(d_{1}, d_{2}\right) \mid l_{1}-l_{2}$ implies $\left(d_{1}, d_{2}\right) \mid l_{1} *-l_{2} *$ since $\left(\left(d_{1}, d_{2}\right), l_{1} l_{2}\right)=1$, whence

$$
k \equiv \frac{\left(l_{1}^{*}-l_{2} *\right)}{\left(d_{1}, d_{2}\right)}{\overline{d_{1}} * l_{1} l_{2}}^{*} \quad\left(\bmod d_{2}{ }^{*} l_{1}{ }^{* *}\right),
$$


or

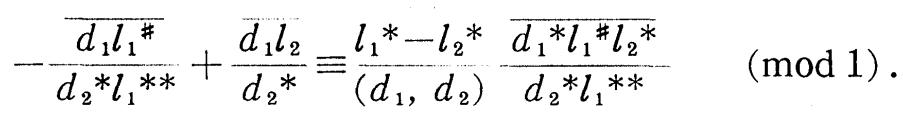

In conjunction with (5.3), (5.4) and (5.5) we finaly obtain

$$
\begin{aligned}
& -\frac{b \bar{e}}{\left[d_{1}, d_{2}\right]} \equiv \frac{a}{e}\left(\frac{1}{d_{1} d_{2} * l_{1}}-\frac{\bar{d}_{1}}{d_{2}{ }^{*} l_{1}}+\frac{\overline{d_{1} l_{2}}}{d_{2} *}\right) \\
& \equiv \frac{a}{e}\left(\frac{1}{d_{1} d_{2}{ }^{*} l_{1}}-\frac{\overline{d_{1} l_{1} \#}}{d_{2} l_{1}{ }^{* *}}-\frac{\overline{d_{1} d_{2}{ }^{*} l_{1}^{* *}}}{l_{1}{ }^{*}}+\frac{\overline{d_{1} l_{2}}}{d_{2}{ }^{*}}\right) \\
& \equiv \frac{a}{e}\left(\frac{1}{d_{1} d_{2}{ }^{*} l_{1}}-\frac{\overline{d_{1} d_{2} * l_{1} * *}}{l_{1}^{\#}}+\frac{l_{1}{ }^{*}-l_{2} *}{\left(d_{1}, d_{2}\right)} \frac{\overline{d_{1}{ }^{*} l_{1} l_{2} l_{2}}}{d_{2}{ }^{*} l_{1}{ }^{* *}}\right) \quad(\bmod 1) \\
& =\frac{a / e}{d_{1} d_{2} * l_{1}}+\theta\left(\frac{d_{1}^{*}}{d_{2} *}\right), \quad \text { say. }
\end{aligned}
$$

We write

$$
\begin{aligned}
a=e f, \quad\left(d_{1}, d_{2}\right) & =\delta, & d_{j}{ }^{*}=\nu_{j}, & \frac{l_{1} *-l_{2} *}{\delta}=l \\
\frac{f Q^{\prime}}{a \delta}+\frac{f}{\delta l_{1}} & =\xi_{2}, & \frac{f Q}{a \delta}+\frac{f}{\delta l_{1}} & =\xi_{1} .
\end{aligned}
$$

By (5.6) and (5.7), (5.1) is reduced to the following:

where

$$
R=\sum_{f \backslash a} \mu\left(\frac{a}{j}\right) \sum_{\substack{l_{1}+l_{2} \leq L \\\left(11 l_{2}, a\right)=1}} \sum_{\substack{\delta l_{1}-l_{2} \\\left(\delta, a l_{1} l_{2}\right)=1}} R_{1}\left(f, l_{1}, l_{2}, \delta\right)
$$

$$
\begin{aligned}
& R_{1}=R_{1}\left(f, l_{1}, l_{2}, \delta\right) \\
& =\sum_{\substack{\left(\nu_{1}, \nu_{2}\right)=1 \\
\left(\nu_{1}, a l_{1}\right)=1 \\
\left(\nu_{2}, a l l_{2}\right)=1}} \lambda_{\delta v_{1}} \lambda_{\delta v_{2}}\left\{\phi\left(\frac{\xi_{2}}{\nu_{1} \nu_{2}}+\theta\left(\frac{\nu_{1}}{\nu_{2}}\right)\right)-\psi\left(\frac{\xi_{1}}{\nu_{1} \nu_{2}}+\theta\left(\frac{\nu_{1}}{\nu_{2}}\right)\right)\right\}
\end{aligned}
$$

with

$$
\begin{aligned}
\theta\left(\frac{\nu_{1}}{\nu_{2}}\right) & =\theta\left(\frac{\nu_{1}}{\nu_{2}}: f, l_{1}, l_{2}, \delta\right) \\
& =-f \frac{\overline{\delta \nu_{1} \nu_{2} l_{1}{ }^{* *}}}{l_{1}{ }^{\#}}+f l \frac{\overline{\nu_{1} l_{1} l_{2}{ }^{*}}}{\nu_{2} l_{1}{ }^{* *}} .
\end{aligned}
$$

and $\xi_{j}$ 's are defined by (5.7).

Next we decompose $\left(\lambda_{\delta \nu}\right)$. Since $\mu^{2}\left(\delta_{\nu}\right)=1$, we may write

$$
\lambda_{\delta \nu}=\sum_{k \leqq(\log D)^{2}} \sum_{\substack{b d=\delta \\ b m \leqq M}} \sum_{\substack{m n=\nu \\ d n \leq N \\ \mu^{2}(n)=1}} a_{b m}(k, M, N) b_{d n}(k, M, N) .
$$

Thus, 


$$
R_{1} \ll(\log D)^{4} \tau(\delta)^{2} \sum_{\substack{M_{1} \\ M_{1}, M_{2} \leq M_{2} \\ M_{1}, N_{2} \leq M}} \sum_{N_{2}} \sup \left|R_{2}\right|
$$

where $M_{1}, N_{1}, M_{2}, N_{2}$ 's run through prowers of 2 ;

$$
\begin{aligned}
R_{2}= & R_{2}\left(M_{1}, N_{1}, M_{2}, N_{2} ; \alpha_{1}, \beta_{1}, \alpha_{2}, \beta_{2}\right) \\
= & \sum_{\substack{\left.m_{1} \sim M_{1} \\
n_{1} \sim N_{1} m_{2} \sim M_{2} n_{2} \sim N_{2} \\
\text { (min } n_{1}, m_{2} n_{2}\right)=1 \\
\left(m_{1} n_{1}, a l 1\right)=\left(n_{2} n_{2}, a l_{2}\right)=1 \\
\mu^{2}\left(n_{1} n_{2}\right)=1}} \alpha_{1}\left(m_{1}\right) \beta_{1}\left(n_{1}\right) \alpha_{2}\left(m_{2}\right) \beta_{2}\left(n_{2}\right)\left\{\phi\left(\frac{\xi_{2}}{m_{1} n_{1} m_{2} n_{2}}+\theta\left(\frac{m_{1} n_{1}}{m_{2} n_{2}}\right)\right)\right. \\
& \left.-\phi\left(\frac{\xi_{1}}{m_{1} n_{1} m_{2} n_{2}}+\theta\left(\frac{m_{1} n_{1}}{m_{2} n_{2}}\right)\right)\right\} ;
\end{aligned}
$$

and the supremum is taken over all sequences $\left(\alpha_{1}\right),\left(\beta_{1}\right),\left(\alpha_{2}\right),\left(\beta_{2}\right)$ such that $\left|\alpha_{1}\right|,\left|\beta_{1}\right|,\left|\alpha_{2}\right|,\left|\beta_{2}\right| \leqq 1$. Moreover

$$
R_{1} \ll(\log D)^{8} \tau(\delta)^{2}\left\{\sup _{\substack{x_{1}, x_{2}, \beta_{1}, \beta_{2} \\ M_{1} N_{1} M_{2} N_{2}>Q x^{-2}}}\left|R_{2}\right|+Q x^{-2 \varepsilon}\right\} .
$$

We shall show

$$
\sup \left|R_{2}\right| \ll Q x^{-3 \varepsilon / 2}
$$

from which Lemma 1 follows. Actually, by (5.9), we then have

$$
\begin{aligned}
R & \ll \sum_{f \backslash a} \sum_{l_{1} \neq l_{2} \leq L} \sum_{\delta \mid l_{1}-l_{2}}(\log D)^{8} \tau(\delta)^{2} Q x^{-3 \varepsilon / 2} \\
& \ll\left(\frac{x}{Q}\right)^{2} x^{\varepsilon} Q x^{-3 \varepsilon / 2} \\
& \ll \frac{x^{2-3 \varepsilon / 2}}{Q} .
\end{aligned}
$$

Combining this with (3.15) we get Lemma 1 .

Now we estimate $R_{2}$ defined by (5.10). By Lemma 2 we have

$$
R_{2}=R_{3}+R_{4}
$$

where

$$
\begin{aligned}
& R_{3}=\sum_{\substack{m_{1} \\
\left(m_{1} n_{1}, m_{2} n_{2}\right)=1}} \sum_{\substack{m_{2} \\
\left(n_{2}\right.}} \frac{\alpha_{1}\left(m_{1}\right) \beta_{1}\left(n_{1}\right) \alpha_{2}\left(m_{2}\right) \beta_{2}\left(n_{2}\right)}{m_{1} n_{1} m_{2} n_{2}} \sum_{0<|h| \leq H} e\left(h \theta\left(\frac{m_{1} n_{1}}{m_{2} n_{2}}\right)\right) \int_{\xi_{1}}^{\xi_{2}} e\left(\frac{h t}{m_{1} n_{1} m_{2} n_{2}}\right) d t, \\
& \left(m_{1} n_{1}, a l_{1}\right)=\left(n_{2} n_{2}, a l_{2}\right)=1
\end{aligned}
$$

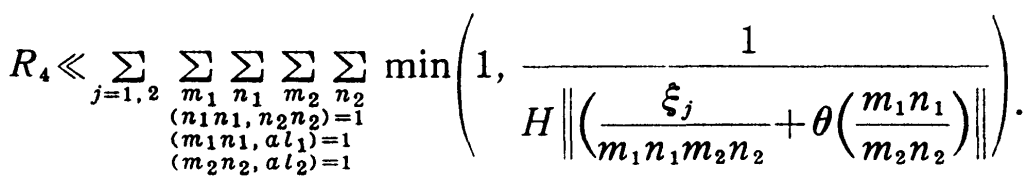

Firstly we consider $R_{4}$. By Lemma 2 


$$
\begin{aligned}
R_{4} & \ll x^{\varepsilon} \sum_{j=1,2} \sum_{\substack{k \sim M_{1} N N_{1}, r \sim M_{2} N_{2} \\
(k, r)=1 \\
(k, a l 1)=\left(r, a l_{2}\right)=1}} \min \left(1, \frac{1}{H \|\left(\frac{\xi_{j}}{k r}+\theta\left(\frac{k}{r}\right) \|\right)}\right) \\
& \ll x^{\varepsilon} \sum_{j=1,2} \sum_{h \in Z}\left|C_{h}\right|\left|\sum_{k} \sum_{r} e\left(\frac{h \xi_{j}}{k r}\right) e\left(h \theta\left(\frac{k}{r}\right)\right)\right| \\
& =x^{\varepsilon} \sum_{j=1,2} \sum_{h \in Z}\left|C_{h}\right||S(h)|, \quad \text { say. }
\end{aligned}
$$

We proceed to estimate $S(h)$. Trivially,

$$
S(h) \ll M_{1} N_{1} M_{2} N_{2} \text {. }
$$

For $h \neq 0$, we get, by partial summation and the definition (5.9) of $\theta$,

$$
\begin{aligned}
& S(h) \ll \sum_{\substack{r \sim M_{2} N_{2} \\
\left(r, a l_{2}\right)=1}}\left(1+\frac{h \xi_{j}}{M_{1} N_{1} r}\right)\left|\sum_{\substack{k \sim M_{1} N_{1} \\
\left(k, r a l_{1}\right)=1}} e\left(h \theta\left(\frac{k}{r}\right)\right)\right| \\
& \ll\left(1+\frac{h Q}{\delta M_{1} N_{1} M_{2} N_{2}}\right) \sum_{\substack{r \sim M_{2} N_{2} \\
\left(r, a l_{2}\right)=1}}\left|\sum_{b=1}^{l_{1} \#} e\left(-h f \frac{\delta k r l_{1}^{* *}}{l_{1}^{\#}}\right) \sum_{\substack{k \sim M 1_{1} N \\
k=b\left(l_{1}\right) \\
\left(k, r a l_{1} * *\right)=1}} e\left(h f l \frac{\overline{k l_{1} \# l_{2}^{*}}}{r l_{1}^{* *}}\right)\right|
\end{aligned}
$$

Lemma 3 yields

$$
\begin{aligned}
& S(h) \ll\left(1+\frac{h Q}{M_{1} N_{1} M_{2} N_{2}}\right) \sum_{\substack{r \sim M_{2} N N_{2} \\
\left(r, a l_{2}\right)=1}} \sum_{b=1}^{l_{1} \#}\left|\sum_{\substack{\left.k \sim M_{2} N_{2} \\
k \equiv b\left(l_{1}\right) \\
k, a r l_{1} * *\right)=1}} e\left(h f l \frac{\overline{k l_{1} \# l_{2}^{*}}}{r l_{1}^{* *}}\right)\right| \\
& \ll x^{\varepsilon / 2}\left(1+\frac{h Q}{M_{1} N_{1} M_{2} N_{2}}\right) \sum_{\left(r, a l_{2}\right)=1} l_{1}^{\#} \tau(a)\left(h f l, r l_{1}^{* *}\right)^{1 / 2}\left(r l_{1}^{* *}\right)^{1 / 2}\left(1+\frac{M_{1} N_{1}}{r l_{1} * *}\right) \\
& \ll \tau(a) x^{\varepsilon / 2}\left(1+\frac{h Q}{M_{1} N_{1} M_{2} N_{2}}\right) l_{1} \sum_{r}(h l, r)^{1 / 2}\left(r^{1 / 2}+\frac{M_{1} N_{1}}{r^{1 / 2}}\right) \\
& \ll \tau(a) x^{\varepsilon / 2}\left(1+\frac{h Q}{M_{1} N_{1} M_{2} N_{2}}\right) l_{1}\left(\sum_{r} \frac{(h l, r)}{r}\right)^{1 / 2}\left\{\left(\sum_{r} r^{2}\right)^{1 / 2}+M_{1} N_{1}\left(\sum_{r} 1\right)^{1 / 2}\right\} \\
& \ll \tau(a) x^{\varepsilon}\left(1+\frac{h Q}{M_{1} N_{1} M_{2} N_{2}}\right) l_{1} \tau(h)\left\{\left(M_{2} N_{2}\right)^{3 / 2}+M_{1} N_{1}\left(M_{2} N_{2}\right)^{1 / 2}\right\},
\end{aligned}
$$

since

$$
\sum_{m \sim M} \frac{(A, m)}{m} \ll \tau(A) .
$$

In conjunction with (5.13), (5.14), (5.15) and Lemma 2, we obtain

$$
\begin{aligned}
R_{4} & \ll x^{\varepsilon} M_{1} N_{1} M_{2} N_{2}\left(\left|C_{0}\right|+\sum_{|h|>H M_{2} N_{2}}\left|C_{h}\right|\right) \\
& +\tau(a) x^{2 \varepsilon} l_{1}\left\{\left(M_{2} N_{2}\right)^{3 / 2}+M_{1} N_{1}\left(M_{2} N_{2}\right)^{1 / 2}\right\} \sum_{0<|h| \leqq H M_{2} N_{2}}\left|C_{h}\right| \tau(h)\left(1+\frac{h Q}{M_{1} N_{1} M_{2} N_{2}}\right)
\end{aligned}
$$




$$
\begin{aligned}
& \ll x^{\varepsilon} M_{1} N_{1} M_{2} N_{2}\left(\frac{\log H}{H}+\sum_{h>H M_{2} N_{2}} \frac{H}{h^{2}}\right)+\tau(a) x^{2 \varepsilon} l_{1}\left\{\left(M_{2} N_{2}\right)^{3 / 2}+M_{1} N_{1}\left(M_{2} N_{2}\right)^{1 / 2}\right\} . \\
& \left.\quad \sum_{0<h \leqq H} \frac{\log H}{H} \tau(h)\left(1+\frac{H Q}{M_{1} N_{1} M_{2} N_{2}}\right)+\sum_{H<h \leqq H N_{2} M_{2}} H \tau(h)\left(\frac{1}{h^{2}}+\frac{Q}{h M_{1} N_{1} M_{2} N_{2}}\right)\right\} \\
& \ll x^{\varepsilon} M_{1} N_{1} M_{2} N_{2}\left(\frac{\log H}{H}+\frac{1}{M_{2} N_{2}}\right) \\
& \quad+\tau(a) x^{2 \varepsilon} l_{1}\left\{\left(M_{2} N_{2}\right)^{3 / 2}+M_{1} N_{1}\left(M_{2} N_{2}\right)^{1 / 2}\right\}(\log H)^{2}\left(1+\frac{H Q}{M_{1} N_{1} M_{2} N_{2}}\right) .
\end{aligned}
$$

Now, we choose

$$
H=\frac{M_{1} N_{1} M_{2} N_{2} X^{4 \varepsilon}}{Q},
$$

then $H>2$ since $M_{1} N_{1} M_{2} N_{2}>Q x^{-2 \varepsilon}$ in (5.11). Thus,

$$
R_{4} \ll x^{\varepsilon}\left(Q x^{-3 \varepsilon}+M_{1} N_{1}\right)+\tau(a) x^{7 \varepsilon} l_{1}\left\{\left(M_{2} N_{2}\right)^{3 / 2}+M_{1} N_{1}\left(M_{2} N_{2}\right)^{1 / 2}\right\}
$$

or

$$
\begin{aligned}
\sup \left|R_{4}\right| & \ll Q x^{-2 \varepsilon}+\tau(a) x^{78}\left(\frac{x}{Q}\right)(M N)^{3 / 2} \\
& \ll Q x^{-2 \varepsilon}+x^{8 \varepsilon}\left(\frac{x}{Q}\right)\left(Q^{3 / 4} x^{-1 / 5-4 \varepsilon}\right)^{3 / 2} \\
& \ll Q x^{-2 \varepsilon}+Q x^{2 \varepsilon}\left(\frac{x^{1 / 5}}{Q^{1 / 4}}\right)^{7 / 2} \\
& \ll Q x^{-3 \varepsilon / 2}
\end{aligned}
$$

We turn to $R_{3}$ defined by (5.12).

$$
\begin{aligned}
& R_{3}=\frac{1}{N_{1} N_{2}} \sum_{\substack{m_{1} \\
\left(n_{1}, m_{2}\right)=1}} \sum_{m_{2}} \alpha_{1}\left(m_{1}\right) \alpha_{2}\left(m_{2}\right) \int_{\xi_{1} / m_{1} m_{2}}^{\xi_{2} / m_{1} m_{2}} \sum_{\substack{\left.m_{1} \\
m_{2} a_{1}\right)=1}} \sum_{\substack{n_{1} \\
\left(m_{1} n_{1}, m_{2} n_{2}\right)=1}} \beta_{1}\left(n_{1}\right) \beta_{2}\left(n_{2}\right) \\
& \begin{array}{cc}
\left(n_{1}, m_{2}\right)=1 & \left(m_{1}\right)=1 \\
\left(m_{1}, a l_{1}\right)=1 & \left(m_{1}, a l_{1} n_{1}, m_{2} m_{2} n_{2}\right)=1 \\
\left(m_{2}, a l_{2}\right)=1 & \mu^{2}\left(n_{1} n_{2}\right)=1
\end{array} \\
& \cdot \frac{N_{1} N_{2}}{n_{1} n_{2}} e\left(\frac{h t}{n_{1} n_{2}}\right) e\left(h \theta\left(\frac{m_{1} n_{1}}{m_{2} n_{2}}\right)\right) d t
\end{aligned}
$$

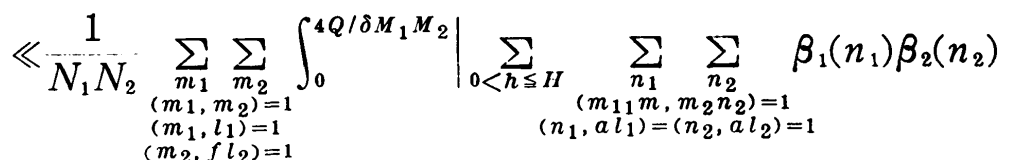

$$
\begin{aligned}
& \cdot \frac{N_{1} N_{2}}{n_{1} n_{2}} e\left(\frac{h t}{n_{1} n_{2}}\right) \cdot e\left(h \theta\left(\frac{m_{1} n_{1}}{m_{2} n_{2}}\right)\right) \mid d t \\
& \ll \frac{Q}{\delta M_{1} N_{1} M_{2} N_{2}} \sup \sum_{m_{1}} \sum_{m_{2}}\left|\sum_{h} \sum_{n_{1}} \sum_{n_{2}} c\left(h, n_{1}, n_{2}\right) e\left(h \theta\left(\frac{m_{11} n}{m_{2} n_{2}}\right)\right)\right|
\end{aligned}
$$


where the suprenum is taken over all sequences $\left|c\left(h, n_{1}, n_{2}\right)\right| \leqq 1$.

In the next section we shall prove the following:

LEMMA 4. For any sequence (c) with $|c|<1$, we have

$$
\begin{aligned}
& S=\sum_{\substack{m_{1} \sim M_{1} \\
\left(m_{1}, m_{2} \sim M_{2}\right)=1 \\
\left(m_{1}, m_{2}\right)=1}}\left|\sum_{0<h \leqq H} \sum_{\substack{n_{1} \sim N_{1} \\
\left(m_{1} n_{1}, m_{2} n_{2} \sim N_{2}\right.}} \sum_{\substack{n_{2} \\
\left(n_{1}, a\right)=1}} c\left(h, n_{1}, n_{2}\right) e\left(h \theta\left(\frac{m_{1} n_{1}}{m_{2} n_{2}}\right)\right)\right|^{2} \\
& \begin{array}{lc}
\left(m_{1}, l_{1}\right)=1 & \left(n_{1}, a l_{1}\right)=\left(n_{2}, a l_{2}\right)=1 \\
\left.m_{2} m_{(n}, f l_{2}\right)=1 & \left.n_{2}\right)=1
\end{array} \\
& \ll x^{\varepsilon} H M_{1} N_{1} M_{2} N_{2}+x^{\varepsilon} l_{1} H^{2}\left(M_{1}+M_{2}\right) M_{2}{ }^{1 / 2} N_{1}{ }^{2} N_{2}{ }^{3} .
\end{aligned}
$$

We apply Lemma 4 to (5.18).

or

$$
\begin{aligned}
R_{3} & \ll \frac{Q}{\delta M_{1} N_{1} M_{2} N_{2}} \sup \left(M_{1} M_{2}\right)^{1 / 2}(S)^{1 / 2} \\
& \ll \frac{x^{4 \varepsilon}}{H}\left\{x^{\varepsilon} H\left(M_{1} M_{2}\right)^{2} N_{1} N_{2}+x^{\varepsilon} l_{1} H^{2}\left(M_{1}+M_{2}\right) M_{1} M_{2}^{3 / 2} N_{1}^{2} N_{2}^{3}\right\}^{1 / 2} \\
& \ll x^{9 \varepsilon / 2}\left\{Q x^{-4 \varepsilon} M_{1} M_{2}+l_{1}\left(M_{1}+M_{2}\right) M_{1} M_{2}^{3 / 2} N_{1}^{2} N_{2}^{3}\right\}^{1 / 2}
\end{aligned}
$$

$$
\begin{aligned}
\sup \left|R_{3}\right| & \ll x^{9 \varepsilon / 2}\left\{Q^{1 / 2} x^{-2 \varepsilon} M+l_{1}^{1 / 2} M^{7 / 4} N^{5 / 2}\right\} \\
& \ll x^{9 \varepsilon / 2}\left\{Q x^{-6 \varepsilon}+\left(\frac{x}{Q}\right)^{1 / 2}\left(Q^{1 / 2} x^{-4 \varepsilon}\right)^{7 / 4}\left(\frac{Q^{1 / 4}}{x^{1 / 5}}\right)^{5 / 2}\right\} \\
& \ll Q x^{-3 \varepsilon / 2} .
\end{aligned}
$$

Combining this with (5.17) we get

as required.

$$
\sup \left|R_{2}\right| \leqq \sup \left|R_{3}\right|+\sup \left|R_{4}\right| \ll Q x^{-3 \varepsilon / 2},
$$

\section{Proof of Lemma 4.}

It remains to establish Lemma 4. By expanding the square and changing the order of summation, we have

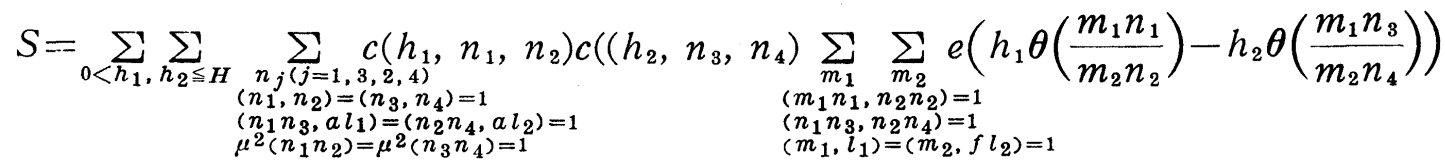

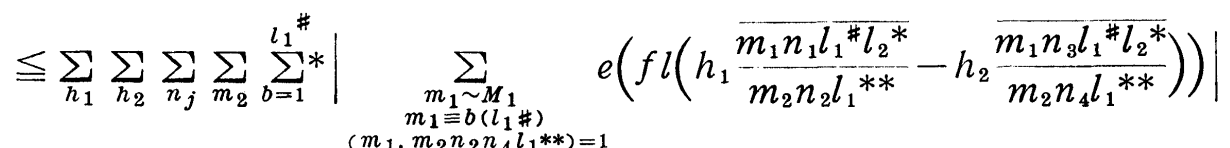

We proceed to treat the argument in the above exponential sums. We have

$$
h_{1} \frac{\overline{m_{1} n_{1} l_{1} l_{2}{ }^{*}}}{m_{2} n_{2} l_{1}{ }^{* *}}-h_{2} \frac{\overline{m_{1} n_{3} l_{1} l_{2} l^{*}}}{m_{2} n_{4} l_{1}{ }^{* *}}=\frac{g}{m_{2} \frac{n_{2}}{\left(n_{2}, n_{3}\right)} \frac{n_{4}}{\left(n_{1}, n_{4}\right)} l_{1}^{* *}}
$$


where

$$
g=h_{1} \frac{1}{\left(n_{2}, n_{3}\right)} \frac{n_{4}}{\left(n_{1}, n_{4}\right)}\left(m_{1} n_{1} l_{1}^{\#} l_{2}{ }^{\prime}\right)^{\prime}-h_{2} \frac{1}{\left(n_{1}, n_{4}\right)} \frac{n_{2}}{\left(n_{2}, n_{3}\right)}\left(m_{2} n_{3} l_{1}^{\#} l_{2}^{*}\right)^{\prime \prime}
$$

with $A^{\prime} A \equiv 1\left(\bmod m_{2} n_{2} l_{1}{ }^{* *}\right)$ and $A^{\prime \prime} A \equiv 1\left(\bmod m_{2} n_{4} l_{1}{ }^{* *}\right)$. We then find, with certain integers $k_{1}$ and $k_{2}$, that

$$
\begin{aligned}
& m_{1} n_{1} n_{3} l_{1}{ }^{*} l_{2}{ }^{*} g \\
& =h_{1} \frac{n_{3}}{\left(n_{2}, n_{3}\right)} \frac{n_{4}}{\left(n_{1}, n_{4}\right)}\left(1+k_{1} m_{2} n_{2} l_{1} * *\right)-h_{2} \frac{n_{1}}{\left(n_{1}, n_{4}\right)} \frac{n_{2}}{\left(n_{2}, n_{3}\right)}\left(1+k_{2} m_{2} n_{4} l_{1} * *\right) \\
& \left.\equiv h_{1} \frac{n_{3}}{\left(n_{2}, n_{3}\right)} \frac{n_{4}}{\left(n_{1}, n_{4}\right)}-h_{2} \frac{n_{1}-\frac{n_{2}}{\left(n_{1}, n_{4}\right)} \quad\left(\bmod m_{2} \frac{n_{2}}{\left(n_{2}, n_{3}\right)}\left(n_{1}, n_{4}\right)\right.}{\left.n_{1}\right)} l_{1}^{* *}\right) \\
& =r, \quad \text { say. }
\end{aligned}
$$

Since $\left(m_{1} n_{1}, m_{2} n_{2}\right)=\left(m_{1} n_{3}, m_{2} n_{4}\right)=1$ and $\mu^{2}\left(n_{1} n_{2}\right)=\mu^{2}\left(n_{3} n_{4}\right)=1$, we have

$$
\left(m_{1} n_{1} n_{3} l_{1}^{*} l_{2}^{*}, m_{2} \frac{n_{2}}{\left(n_{2}, n_{3}\right)} \frac{n_{4}}{\left(n_{1}, n_{4}\right)} l_{1}^{* *}\right)=1,
$$

whence

$$
g \equiv \overline{m_{1} n_{1} n_{3} l_{1}^{\#} l_{2}{ }^{*}} r \quad\left(\bmod m_{2} \frac{n_{2}}{\left(n_{2}, n_{3}\right)} \frac{n_{4}}{\left(n_{1}, n_{4}\right)} l_{1}^{* *}\right) .
$$

Now Lemma 3 yields

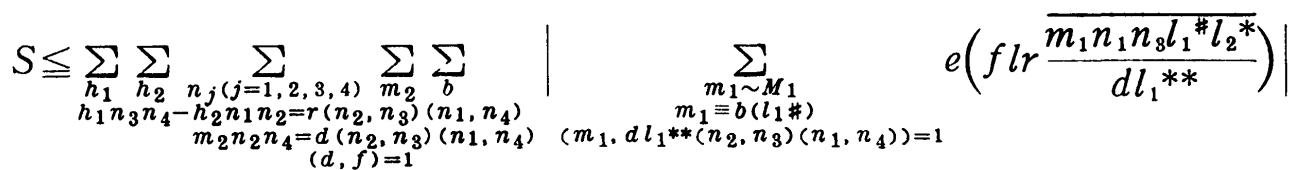

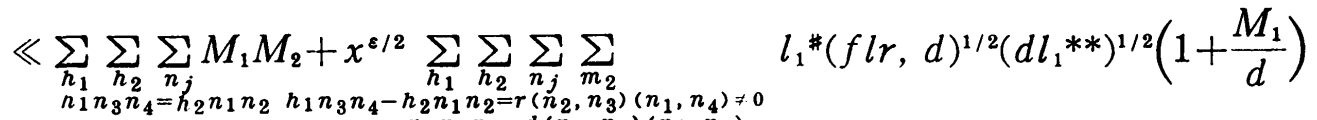

$$
\begin{aligned}
& m_{2} n_{2} n_{4}=d\left(n_{2}, n_{3}\right)\left(n_{1}, n_{4}\right) \\
& (d, f)=1 \\
& \ll M_{1} M_{2} \sum_{s \leqq 1 N_{1} N_{2}} \tau_{3}(s)^{2}+x^{\varepsilon / 2} l_{1} \sum_{n_{1}, h_{2}}\left(\sum_{n_{j}} \sum_{m_{2}} \frac{(k r, d)}{d}\right)^{1 / 2}\left\{\left(\sum_{n_{j}} \sum_{m_{2}} d^{2}\right)^{1 / 2}+M_{1}\left(\sum_{n_{j}} \sum_{m_{2}} 1\right)^{1 / 2}\right\} \\
& \ll x^{\varepsilon} H M_{1} N_{1} M_{2} N_{2}+x^{\varepsilon / 2} l_{1} \sum_{h_{1}, h_{2}}\left(\sum_{n_{j}} \sum_{m_{2}} \frac{(k r, d)}{d}\right)^{1 / 2} \\
& \left\{\left(\left(M_{2} N_{2}^{2}\right)^{3} N_{1}^{2}\right)^{1 / 2}+M_{1}\left(M_{2} N_{2}^{2} N_{1}^{2}\right)^{1 / 2}\right\} \text {. }
\end{aligned}
$$

Here we easily see

$$
\Sigma=\sum_{n_{j}} \sum_{m_{2}} \frac{(k r, d)}{d} \ll x^{\varepsilon} N_{1}^{2}
$$

In fact, if we write

$$
\begin{array}{lll}
\delta_{1}=\left(n_{1}, n_{2}\right), & \delta_{2}=\left(n_{2}, n_{3}\right) \\
n_{1}=\delta_{1} n_{1}^{\prime}, \quad n_{2}=\delta_{2} n_{2}^{\prime}, \quad n_{3}=\delta_{2} n_{3}^{\prime}, \quad n_{4}=\delta_{1} n_{4}^{\prime},
\end{array}
$$


and

then we have

$$
\gamma=\left(n_{2}^{\prime}, n_{4}^{\prime}\right), \quad n_{2}^{\prime}=\gamma n_{2}, \quad n_{4}^{\prime}=\gamma n_{4}
$$

$$
\begin{aligned}
\sum & =\sum_{\substack{n_{1} \\
n_{1}}} \sum_{n_{3}} \sum_{\left.\delta_{1} \mid n_{1} n_{3}^{\prime} n_{4}-h_{2} n_{1}^{\prime} n_{1}^{\prime} n_{2}\right) \neq 0} \sum_{\substack{n_{2} \\
m_{2}}} \sum_{\substack{\gamma \\
\left(n_{2}, n_{4}\right)=1}} \sum_{\substack{n_{2} \\
n_{n_{4}}}} \frac{\left(k\left(h_{1} n_{3}^{\prime} n_{4}-h_{2} n_{1}^{\prime} n_{2}\right), m_{2} \gamma n_{2} n_{4}\right)}{m_{2} \gamma n_{2} n_{4}} \\
& \leqq \sum_{n_{1}} \sum_{n_{3}} \sum_{\delta_{1}} \sum_{\delta_{2}} \sum_{n_{2}} \sum_{n_{4}} \sum_{m_{2}} \sum_{\gamma} \frac{\left(B, m_{2}\right)}{m_{2}} \frac{(B, \gamma)}{\gamma} \frac{\left(B, n_{2}\right)}{n_{2}} \frac{\left(B, n_{4}\right)}{n_{4}}
\end{aligned}
$$

where $B=k\left(h_{1} n_{3}^{\prime} n_{4}-h_{2} n_{1}^{\prime} n_{2}\right) \neq 0$. Since $\left(n_{2}, n_{4}\right)=1$,

and

$$
\left(B, n_{2}\right)=\left(k h_{1} n_{3}^{\prime} n_{4}, n_{2}\right)=\left(k h_{1} n_{3}^{\prime}, n_{2}\right)
$$

By (5.16),

$$
\left(B, n_{4}\right)=\left(k h_{2} n_{1}^{\prime}, n_{4}\right) \text {. }
$$

$$
\begin{aligned}
\Sigma & \ll\left(x^{\varepsilon / 5}\right)^{2} \sum_{n_{1}} \sum_{n_{3}} \sum_{\delta_{1} \mid n_{1}} \sum_{\delta_{2} \mid n_{3}} \sum_{n_{2}} \frac{\left(k h_{1} n_{3}^{\prime}, n_{2}\right)}{n_{2}} \sum_{n_{4}} \frac{\left(k h_{2} n_{1}^{\prime}, n_{4}\right)}{n_{4}} \\
& \ll\left(x^{\varepsilon / 5}\right)^{4} \sum_{n_{1}} \sum_{n_{3}} \tau\left(n_{1}\right) \tau\left(n_{3}\right) \\
& \ll x^{8} N_{1}^{2} .
\end{aligned}
$$

Therefore we get

$$
\begin{aligned}
S & \ll x^{8} H M_{1} N_{1} M_{2} N_{2}+x^{\varepsilon} l_{1} H^{2}\left\{M_{2}^{3 / 2}{N_{1}}^{2} N_{2}{ }^{3}+M_{1} M_{2}^{1 / 2} N_{1}^{2} N_{2}\right\} \\
& \ll x^{8} H M_{1} N_{1} M_{2} N_{2}+x^{\varepsilon} l_{1} H^{2}\left(M_{1}+M_{2}\right) M_{2}^{1 / 2} N_{1}^{2} N_{2}^{3},
\end{aligned}
$$

as required.

This conpletes the proof of our Theorem.

\section{References}

[1] Fouvry, E., Autour du théorème de Bombieri-Vinogradov, Acta Math. 152 (1984), 219-244.

[2] Fouvry, E., Théorème de Brun-Titchmarsh; application au théorème Fermat, Invent. Math. 79 (1985), 383-407.

[3] Fouvry, E., Autour du théorème de Bombieri-Vinnogradov. II, Ann. scient. Éc. Norm. Sup. 20 (1987), 617-640.

[4] Hooley, C., On the Brun-Titchmarsh theorem. II, Proc. London Math. Soc. (3), 30 (1975), 114-128.

[5] Hooley, C., Applications of sieve methods to the theory of numbers, Cambridge 1976.

[6] Iwaniec, H., A new form of the error term in the linear sieve, Acta Arith. 37 (1980), 307-321.

[7] Iwaniec, H., On the Brun-Titchmarsh theorem, J. Math. Soc. Japan 34 (1982), 95123. 
[8] Mikawa, H., Almost-primes in arithmetic progressions and short intervals, Tsukuba J. Math. 13 (1989), 387-401.

[9] Motohashi, Y., On almost-primes in arithmetic progressions, II, Proc. Japan Acad. 51 (1975), 545-547.

[10] Richert, H.E., Selberg's sieve with weight, Mathematika 16 (1969), 545-547.

Institute of Mathematics

University of Tsukuba 